Apidologie, 1973, 4 (1), 81-85.

\title{
VERBREITUNG UND POLLENANALYTISCHE BEDEUTUNG DER SIBBERURZ (DRYAS OCTOPETALA)
}

Dryas octopetala : répartition et intérêt mélisso-palynologique

Annemarie FOSSEL

A-8943 Aigen im Ennstal, Österreich

SUMMARY

DISTRIBUTION AND POLLEN ANALYTIC IMPORTANCE OF DRYAS OCTOPETALA

Distribution, floral structure, the pollen and insect visits of Dryas octopetala (Geum octopetalum) (Rosaceae) are described. The occurence of single Dryas pollen grains in Austrian alpine honeys allows an exact geographical determination of their origin. As Dryas octopetala is growing only on calcareous and dolomitic soil they originate from the higher sites of the Kalkalpen.

\section{ZUSAMMENFASSUNG}

Verbreitung, Blütenbau und Insektenbeflug der Silberwurz (Dryas octopetala; Fam. Rosaceae) sowie ihr Pollen werden beschrieben. Einzelfunde von Dryaspollen in österreichischen Alpenhonigen gestatten eine genaue pflanzengeografische Herkunftsbestimmung aus höheren Lagen der Kalkalpen, da die Silberwurz nur auf Kalk und Dolomit verbreitet ist.

In den Alpen gedeiht die Silberwurz, Dryas octopetala, aus der Familie der Rosaceae ausschließlich in höheren Lagen der Kalkalpen, so daß Dryas 
pollen im Honigbild eindeutig auf eine Tracht aus der alpinen Region der nördlichen und südlichen Kalkalpen hinweist und damit auch eine nähere Determination der Rhododendronpollen gestattet, von denen in den Alpen nur Rhododendron hirsutum kalkstet ist.

\section{STANDORT UND VERBREITUNG}

Die Silberwurz, Dryas octopetala, ist als Spalierstrauch mit ihrer langen, dünnen Pfahlwurzel, mit ihrem niedrigen Wuchs und ihren ledrigen, immergrünen Laubblättern ein vorzüglich ausgerüsteter $P$ flanzenpionier, dem es gelingt, auf bewegten, steilen Schutt- und Blockhalden festen Halt zu finden und als erste Blütenp flanze Moränen, Alluvionen und Kare zu besiedeln.

Die weißfilzige Blattunterseite, die wie Silber glänzt, hat dem dichtbelaubten Spalierstrauch den Namen gegeben.

Die dunkelgrünen, gesägten Blätter bilden in Höhen von $1000-2500 \mathrm{~m}$ auf Kalkunterlage oft große, geschlossene Polster, sogenannte « SilberwurzRasen 》. Fossile Blattreste beweisen, daß die Silberwurz vermutlich während der letzten Eiszeit über die Karpathen in die Alpen eingewandert ist.

Heute ist die Art zirkumpolar verbreitet; sie gehört den arktisch-alpinen Florenelementen an und ist eine der am weitesten nordwärts reichenden Blütenp flanzen. In ihren Bodenansprüchen ist sie ausgesprochen kalkfordernd, gedeiht daher in den Alpen ausschließlich auf Kalk und Dolomit, auf Grobund Feinschutt und auf Alpenhumus in der Zwergstrauchheide an der oberen Vegetationsgrenze. Im geschlossenen Rasen vermag sie sich nicht zu halten, nur im Bach- und Lavinengeröll steigt sie gelegentlich bis auf $1000 \mathrm{~m}$ oder etwas tiefer herab.

\section{BLÜTENBAU UND INSEKTENBESUCH}

Je nach der Höhenlage schmücken sich die Silberwurzposlter von Mai bis August dicht mit zarten, weißen Blüten, die den Brombeerblüten ähnlich sehen. Die Blüten besitzen $7-9$ Kelchblätter und ebensoviele reinweiße Kronblätter. Die zahlreichen Staubblätter tragen gelbe Staubbeutel. Der Nektar wird wie bei den verwandten Brombeerblüten von einer am Grunde der Blüte gelegenen Scheibe (Diskus) abgesondert und ist allen Besuchern leicht zugänglich.

Die Blüten werden von Fliegen besucht; es stellen sich aber auch Käfer und Schmetterlinge ein und können die Bestäubung vollziehen. Die pollenreichen Blüten sind auch für Honigbienen sehr anziehend, man kann die Sammlerinnen bei einigermaßen gutem Flugwetter bis in extreme Höhenlagen der Alpen als regelmäßige und eifrige Besucher der Silberwurz beobachten. 
Die zahlreichen Fliegen scheinen den Bienen wenig Konkurrenz zu machen, Man kann immer wieder sehen, daß die Bienen die Blüten trotz des eben stattgefundenen Fliegenbesuches ausgiebig bearbeiten, indem sie Kopf und Rüssel mehrmals an verschiedenen Stellen zwischen die Staubgefäße versenken. Sie bepudern sich dabei Kopf und Bauch mit gelbem Blütenstaub und sammeln oft große Pollenhöschen.

\section{BIENENWIRTSCHAFTLICHE BEDEUTUNG}

Durch die weiten Flugstrecken, die die Honigbienen von den Bienenständen im Tale zu den Silberwurzrasen auf den steilen Schuttkegeln der Kalkfelsen zurücklegen müssen, ist der bienenwirtschaftliche Wert dieser Tracht leider ganz unbedeutend. Von höher gelegenen Wanderständen aus sind die Silberwurzbestände den Sammlerinnen leichter erreichbar, und es sind daher Einzelpollen von Dryas octopetala in den Honigen aus den österreichischen Kalkalpen keine Seltenheit. Sie treten sehr regelmäßig in allen Prohen auf, die Erica-carnea- und Rhododendron-pollen als Begleit- oder Leitpollen enthalten.

Besonders in den nördlichen Kalkalpen ist Dryas octopetala von den niederösterreichischen Ausläufern der Alpen (Rax, Schneeberg) bis nach Tirol in Frühjahrs- und Sommerhonigen als charakteristicher Einzelpollen vertreten und gestattet dadurch, die Alpenrosenhonige der Zentralalpen von denen aus den Kalkalpen mittels der Honig-Pollenanalyse zu unterscheiden.

Pollen von Rhododendron ferrugineum und $R$. hirsutum lassen sich im Pollenbild wegen ihrer großen Ahnlichkeit im allgemeinen nicht trennen, bei Anwesenheit von Dryas octopetala läßt sich aber mit Sicherheit auf Tracht von der rauhhaarigen, kalkholden Art Rh. hirsutum schließen.

Durch diesen pflanzengeographischen Hinweis ist der Pollen von Dryas octopetala im Honigbild der Alpenhonige sehr aufschlußreich und sollte mehr als bisher in der Honig-Pollenanalyse Beachtung finden.

Auch in den nordeuropäischen Honigen ist das Vorkommen von Dryaspollen sehr wahrscheinlich.

\section{BESChREIbUNG DES POLLENS}

Pollenprobe A. Fossel, Mai 1971, Präparat Hohenheim, Februar 1972. Dryas octopetala liefert kleine bis mittelgroße, blaß grünlichegelbe, isopolare Pollenkörner. Die Polansicht ist abgerundet dreiseitig, die Seitenansicht breitoval bis circulär. Das PK hat 3 Falten mit mehr oder weniger ausgebildeter Poroide. Die Falte wird von zahlreichen Exineresten bedeckt; Länge der Falte 

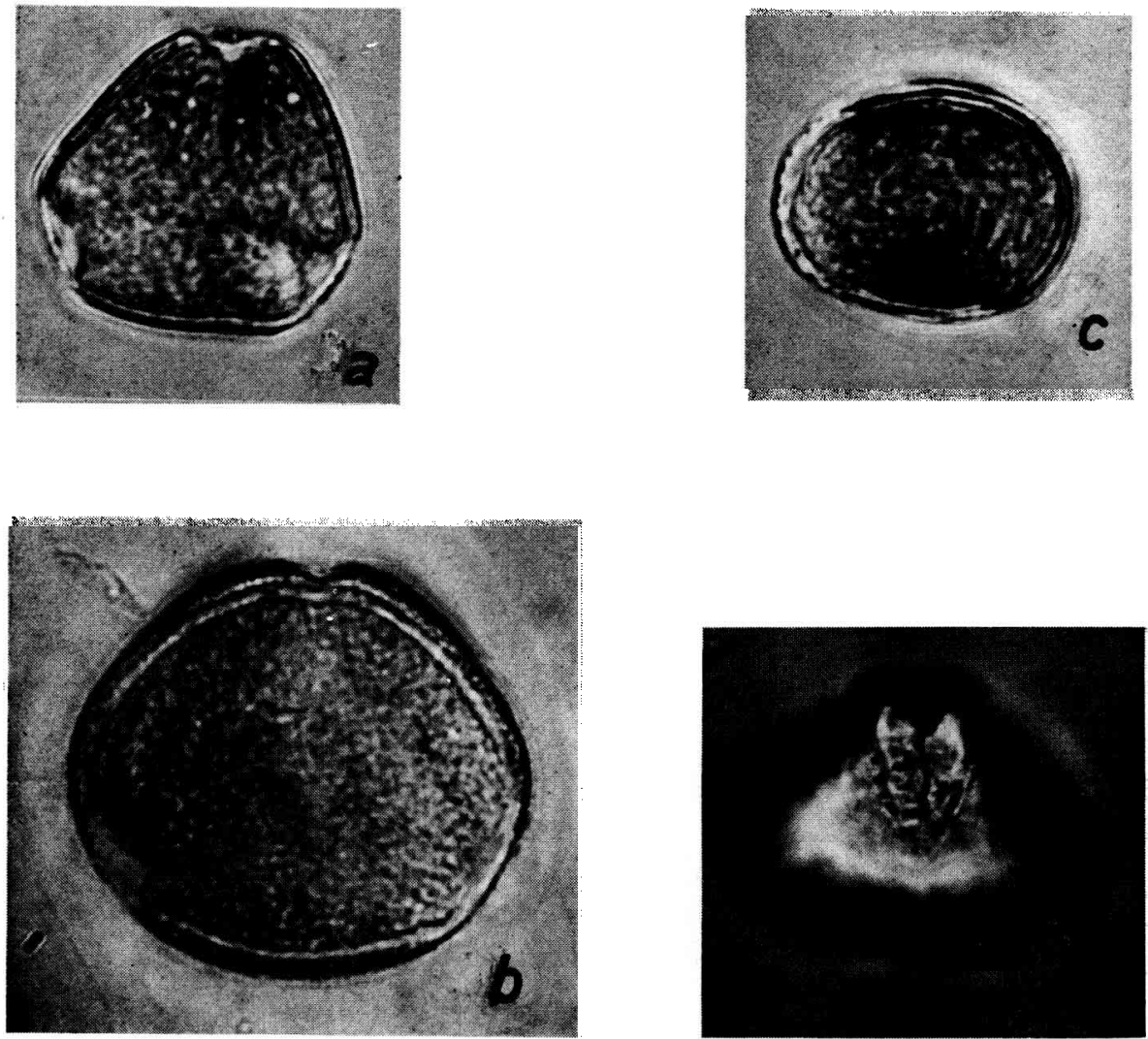

Aвв. 1. - Pollen von Dryas octopetala.

a) Pollage, optischer äquatorialer Schnitt, $1000 \mathrm{x}$.

b) Pollage, optischer äquatorialer Schnitt, Pollenkorn weniger stark gequollen als bei a). Vergrößerung $1400 \mathrm{x}$; bei dieser stärkeren Vergrößerung erscheint die rugulate Exineskulptur im optischen Schnitt als feine Querstrichelung.

c) Seitenlage, optischer meridionaler Schnitt.

d) Schiefe Pollage, Aufsicht auf die Falte.

Fig. 1. - Pollen de Dryas octopetala

a) Vue polaire, coupe optique équatoriale, $1000 \mathrm{x}$

b) Vue polaire, coupe optique équatoriale, grain de pollen moins fortement gonflé qu'en a). Grossissement $1400 \mathrm{x}$; à ce plus fort grossissement la sculpture rugulée de l'exine apparaît en coupe optique comme finement striée.

c) Vue latérale, coupe optique méridienne

d) Vue polaire inclinée; on voit le sillon

ca. $20 \mu$, Breite am Aquator ca. $12 \mu$, Faltenrand leicht aufgwölbt, nicht ganz regelmäßig. Faltenenden mäßig spitz zulaufend, Abstand der Faltenenden am Pol ca. $5 \mu$.Die Exine ist $1 \mu$ dick und zeigt eine sehr feine zarte kurze Strichelung (kurz-rugulate Skulptur); Dicke der Intine $1 \mu$. 
Bei der Messung von 50 Pollenkörnern ergab sich ein Durchschnittswert für die Polachse von 29,4 $\mu$, die Aquatorialachse ist gleichlang.

Tricolpate und tricolporoidate PK sind in der Familie der Rosaceen weitverbreitet. Dryas ist also keine hochcharakteristische Form. Entsprechend muß bei der Bestimmung besonders kritisch vorgegangen werden.

Eingegangen im November 1972.

Reçu pour publication en novembre 1972.

\section{DANKSAGUNG}

Die Pollenbeschreibung und die Abbildungen des Dryas-Pollens verdanke ich Herrn Dr. G. VorwoHL, Stuttgart-Hohenheim.

\section{RESUME}

Dryas octopetala, Rosacée, appartient à la flore arcto-alpine et est l'un des Phanérogames qui pénètrent le plus profondément vers le Nord. Dans les Alpes elle ne prospère que sur les terrains calcaires et dolomitiques où elle forme, à la limite supérieure de la végétation, dans les éboulis fin ou grossiers, des coussins étendus qui, selon l'altitude se couvrent de mai à août de délicates fleurs blanches qui offrent aux abeilles pollen et nectar.

Dans les miels des Alpes calcaires d'Autriche du nord le pollen de Dryas octopetala est un pollen isolé caractéristique et suffisamment fréquent pour que sa présence permette de faire la distinction entre les miels de Rhododendron des Alpes centrales et ceux des Alpes calcaires. Il est très probable que le pollen de Dryas se trouve également dans les miels du nord de l'Europe.

\section{LITERATUR}

Hegr D., 1935. Illustrierte Flora Mitteleuropas. 2. Auflage, B IV/2, S. 922-927. Hansaverlag, München. 